\title{
Systematics of a widely distributed western North American springsnail, Pyrgulopsis micrococcus (Caenogastropoda, Hydrobiidae), with descriptions of three new congeners
}

\author{
Robert Hershler ${ }^{1, \dagger}$, Hsiu-Ping Liu ${ }^{2, \ddagger}$, Corbin Bradford ${ }^{2, \S}$
}

I Department of Invertebrate Zoology, Smithsonian Institution, P.O. Box 37012, Washington, DC 200137012, USA 2 Department of Biology, Metropolitan State University of Denver, Denver, CO 80217, USA

† http://www.zoobank.org/8448C2D3-FC14-4590-A07C-0C2BA402C1B0

$\ddagger$ http://www.zoobank.org/ADD77566-363F-448F-8F52-552A173E90E7

§ http://www.zoobank.org/2037981F-B729-452A-B321-C925300199E0

Corresponding author: Robert Hershler (hershlerr@si.edu)

Academic editor: Eike Neubert | Received 24 June 2013 | Accepted 26 August 2013 | Published 10 September 2013

http://www.zoobank.org/64BCF75A-87C9-4302-B4B4-CEC439450805

Citation: Hershler R, Liu H-P, Bradford C (2013) Systematics of a widely distributed western North American springsnail, Pyrgulopsis micrococcus (Caenogastropoda, Hydrobiidae), with descriptions of three new congeners. ZooKeys 330: 27-52. doi: 10.3897/zookeys.330.5852

\begin{abstract}
We describe three new species of springsnails (genus Pyrgulopsis) from the Amargosa River basin, California and Nevada (P. licina sp. n., P. perforata sp. n., P. sanchezi sp. n.), each of which was previously considered to be part of $P$. micrococcus. We also restrict $P$. micrococcus to its type locality area (Oasis Valley) and redefine a regional congener, $P$. turbatrix, to include populations from the central Death Valley region and San Bernardino Mountains that had been previously identified as $P$. micrococcus. The five species treated herein form genetically distinct lineages that differ from each other by $4.2-12.6 \%$ for $\mathrm{mtCOI}$ and $5.2-13.6 \%$ for mtNDI (based on previously published and newly obtained data), and are diagnosable by shell and/or penial characters. The new molecular data presented herein confirm sympatry of P. licina and $P$. sanchezi in Ash Meadows (consistent with morphological evidence) and delineate an additional lineage of P. micrococcus (in the broad sense) that we do not treat taxonomically owing to the paucity of morphological material. Conservation measures are needed to ensure the long term persistence of populations of P. micrococcus and a genetically differentiated lineage of $P$. sanchezi which live in disturbed habitats on private lands.
\end{abstract}

\section{Keywords}

Pyrgulopsis, Hydrobiidae, Gastropoda, United States, California, Nevada, freshwater, taxonomy, conservation

Copyright Robert Hershler et al. This is an open access article distributed under the terms of the Creative Commons Attribution License 3.0 (CC-BY), which permits unrestricted use, distribution, and reproduction in any medium, provided the original author and source are credited. 


\section{Introduction}

The western North American hydrobiid gastropod genus Pyrgulopsis (commonly known as springsnails) is composed of 134 currently recognized species (Hershler and Liu 2012) which typically live in springs and have very narrow geographic ranges. This large radiation is characterized by a high degree of morphological conservatism and homoplasy, which has posed difficulties in delineating species limits and phylogenetic relationships (Hershler 1994, Liu and Hershler 2005). Although the recent use of molecular tools has facilitated considerable progress in establishing a species level taxonomy that reflects the phylogenetic history of Pyrgulopsis (e.g., Hershler et al. 2003, Hershler and Liu 2004, Hershler and Liu 2009), a number of issues have yet to be addressed, including the unsettled status of several widely ranging congeners which have been shown to be composites of genetically divergent lineages (Hurt 2004, Liu et al. 2003, Liu and Hershler 2007). There is an urgent need to resolve these taxonomic problems to help identify conservation priorities for Pyrgulopsis, which is a current focus of attention of land managers owing to the increasing threats to its groundwater-dependent habitats (e.g., USFWS 2012a-c).

Pyrgulopsis micrococcus (Pilsbry in Stearns, 1893) was originally described based on shells from two localities in the Amargosa River basin and early treated as endemic to the upper portion of this watershed (Gregg and Taylor 1965, Taylor 1985: 317). This species was subsequently revised to include additional populations scattered within large portions of the Mojave Desert (southeastern California and southwestern Nevada) that resembled specimens from the type locality area in having a globose to ovate-conic shell and distally lobate penis with a terminal gland (sometimes reduced or absent) on the ventral surface (Hershler and Sada 1987, Hershler 1989, Hershler and Pratt 1990). A recent phylogenetic analysis resolved mtDNA sequences from 29 populations of $P$. micrococcus into five deeply divergent, allopatric clades-one of which also included morphologically similar and geographically proximate $P$. turbatrix Hershler, 1998-which were postulated to be distinct species (Liu et al. 2003, also see Hershler and Liu 2008). In this paper we detail previously unrecognized shell and penial differences supporting recognition of three of these lineages as new species which we describe herein while also clarifying the limits of $P$. micrococcus and $P$. turbatrix. We also present additional molecular data that confirm sympatry of two of these novelties at various sites in Ash Meadows (consistent with our morphological evidence) and delineate a new lineage of $P$. micrococcus (in the broad sense) in northern Death Valley that is not taxonomically treated owing to inadequate material.

\section{Methods}

The previous phylogeographic investigation (Liu et al. 2003) was based on sampling across the entire broad range of $P$. micrococcus, including each of the drainage basins inhabited by this species. For the current study additional molecular sampling was done to confirm the apparent sympatry of two P. micrococcus lineages at various sites in Ash 
Meadows that was discovered during the course of this taxonomic study, and to evaluate the relationships of a distinctive morphotype (of P. micrococcus) in Grapevine Springs (northern Death Valley) that was not included in our previous analysis. Several previously analyzed populations-Grapevine Springs (M2), Purgatory Spring (M8), Tecopa Spring (M25), Shoshone Spring (M26)-were also additionally sampled to increase sample size and further evaluate their genetic distinctiveness. Newly collected material was preserved in $90 \%$ ethanol in the field. Genomic DNA was extracted from entire snails (2-10 specimens per sample) using a CTAB protocol (Bucklin 1992); each specimen was analyzed for mtDNA individually. LCO1490 and HCO2198 (Folmer et al. 1994) were used to amplify a 710 base pair (bp) fragment of COI, and ND43F and RND592F (Liu et al. 2003) were used to amplify a $550 \mathrm{bp}$ fragment of NADH dehydrogenase subunit I (NDI). Amplification conditions and sequencing of amplified polymerase chain reaction product followed Liu et al. (2003). Sequences were determined for both strands and then edited and aligned using SEQUENCHERä version 5.0.1. The 51 newly sequenced specimens were analyzed together with our previously published $P$. micrococcus dataset (Liu et al. 2003, Hershler and Liu 2008). The new haplotypes from each sampling locality were deposited in GenBank (accession numbers KF559184-KF559202). Sample information and GenBank accession numbers are given in Appendix I. One example of each haplotype detected in a given sample was used in our analyses.

The partition homogeneity/incongruence length difference test (Farris et al. 1994) was used to determine whether the COI and NDI datasets were consistent and could be combined for the phylogenetic analysis. The test, which was conducted using parsimony-informative sites only and 1,000 replicates, indicated no significant incongruence $(P=0.36)$ and consequently we combined the two datasets in our phylogenetic analysis. MRMODELTEST 2.3 (Nylander 2004) was used to obtain an appropriate substitution model (using the Akaike Information Criterion) and parameter values for this analysis. Phylogenetic relationships were inferred by Bayesian analysis using MRBAYES 3.1.2 (Huelsenbeck and Ronquist 2001). Metropolis-coupled Markov chain Monte Carlo simulations were run with four chains (using the model selected through MRMODELTEST) for 5,000,000 generations, and Markov chains were sampled at intervals of 10 generations to obtain 500,000 sample points. We used the default settings for the priors on topologies and the GTR + I + G model parameters selected by MRMODELTEST as the best fit model. At the end of the analysis, the average standard deviation of split frequencies was less than 0.01 (0.0063) and the Potential Scale Reduction Factor (PSRF) was 1 , indicating that the runs had reached convergence. The sampled trees with branch lengths were used to generate a $50 \%$ majority rule consensus tree with the first $25 \%$ of the samples removed to ensure that the chain sampled a stationary portion. Genetic distances (maximum composite likelihood) within and between species/lineages were calculated using MEGA5 (Tamura et al. 2011), with standard errors estimated by 1,000 bootstrap replications with pairwise deletion of missing data.

Types and other voucher material were deposited in the National Museum of Natural History (USNM) collection. Relevant material from the Academy of Natural Sciences of Philadelphia (ANSP), Bell Museum of Natural History (BellMNH) and 
the Santa Barbara Museum of Natural History (SBMNH) was also examined during the course of this study. Series of large adults $(n=10)$ were used for shell measurements. Whorl counts refer to the entire shell. Sexual dimorphism in shells, which is occasionally observed in Pyrgulopsis (Taylor 1987), could not be quantified owing to small sample sizes. The total number of shell whorls was counted (WH) for each specimen; and the height and width of the entire shell (SH, SW), body whorl (HBW, WBW), and aperture (AH, AW) were measured from camera lucida outline drawings using a digitizing pad linked to a personal computer (see Hershler 1989). In addition, three ratios were generated from the raw data (SW/SH, HBW/SH, AH/SH). Descriptive statistics were generated using SYSTAT FOR WINDOWS 11.00.01 (SSI 2004). Penial variation was described from series of adult specimens (typically $n=30$ ) that were relaxed with menthol crystals and fixed in dilute formalin prior to preservation in $70 \%$ ethanol. Descriptive penial terminology is from Taylor (1987) and Hershler (1994, $1998)$. Variation in the number of cusps on the radular teeth $(n=5)$ was assessed using the method of Hershler et al. (2007).

We used a conservative, evolutionary lineage concept in describing new species only for those snails that are morphologically diagnosable as well as phylogenetically independent and substantially divergent genetically (Hershler et al. 2007). Inasmuch as the principal goal of our paper is to delimit species, we provide only brief taxonomic descriptions which focus on those aspects of morphology that have proven most useful in previous such studies of Pyrgulopsis (Taylor 1987, Hershler 1994, Hershler 1998).

\section{Results}

The alignment of COI and NDI sequences yielded $1188 \mathrm{bp}$. The five previously reported clades (A-E) were similarly recovered in the Bayesian analysis of this combined dataset (Fig. 1). The P. micrococcus morphotype in Grapevine Springs which was not included in our prior analysis formed an additional lineage (F) together with specimens from a spring in the Southern California coastal drainage. This clade is not formally treated herein owing to the paucity of morphological material. The additional molecular sampling conducted for this study also confirmed sympatry of morphologically distinctive clades $\mathrm{C}$ and $\mathrm{E}$ at three localities in Ash Meadows (M51-52, M53-54, M57-58) (Fig. 1), providing additional support for recognizing these as separate species.

Clades A-F differed from each other by $4.2-12.6 \%$ for COI and $5.2-13.6 \%$ for NDI; variation within clades ranged from $0-2.5 \%$ for COI and $0-3.5 \%$ for NDI (Appendix II). The geographic distributions of these genetic lineages are shown in Fig. 2. Based on the genetic evidence of distinctiveness and diagnosable shell and/or penial characters (detailed below) we recognize three of these lineages as new species which are described below (clade $\mathrm{B}$ as $P$. perforata, clade $\mathrm{C}$ as $P$. licina, clade $\mathrm{E}$ as $P$. sanchezi), restrict $P$. micrococcus to its type locality area (Oasis Valley, clade A), and revise $P$. turbatrix to include populations from the central Death Valley region and San Bernardino Mountains that had been previously identified as P. micrococcus (clade D). 


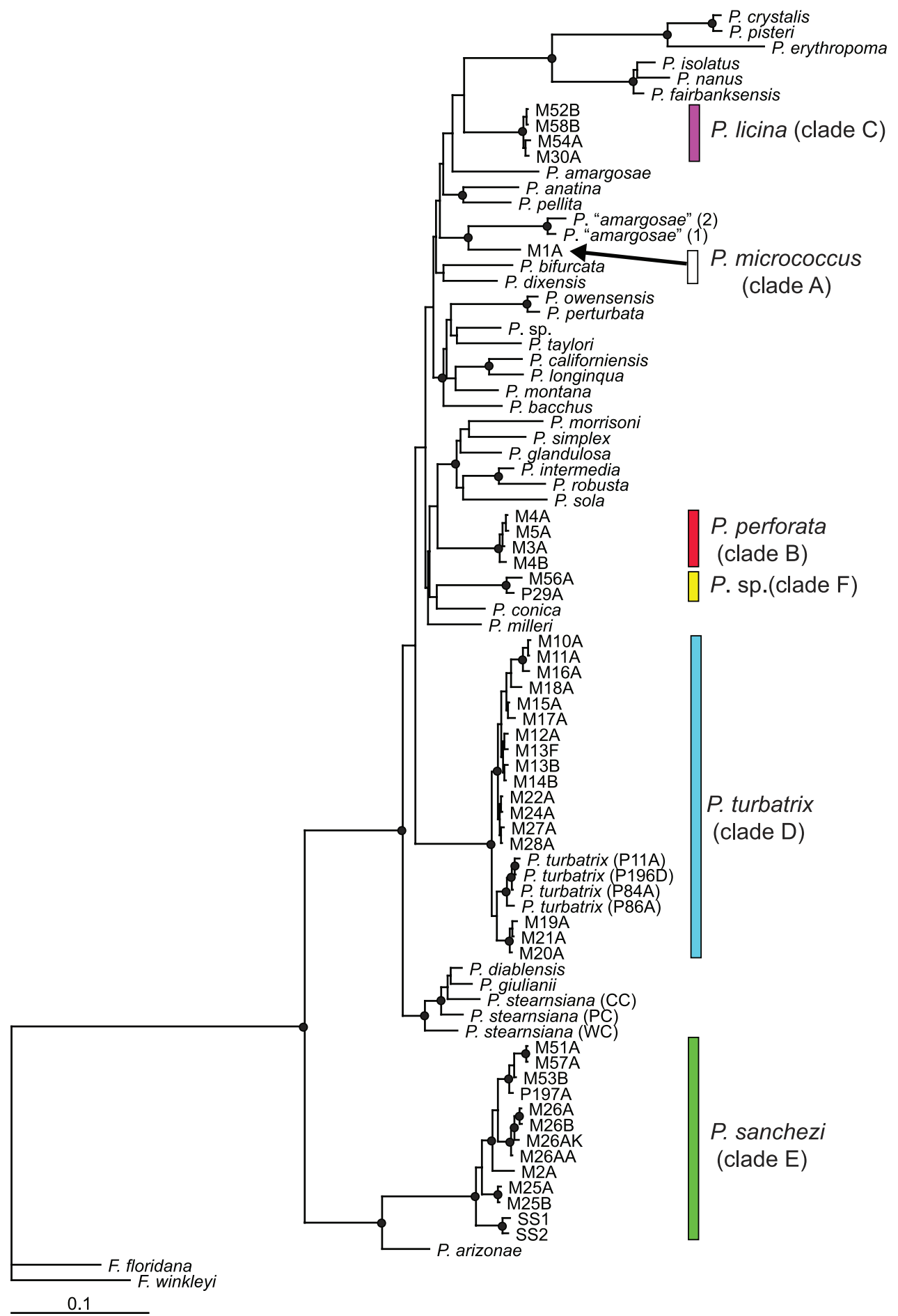

Figure I. Bayesian tree based on the combined (COI, NDI) dataset. Nodes having posterior probabilities $>95 \%$ are identified by filled circles. Specimen codes are from Appendix I. 


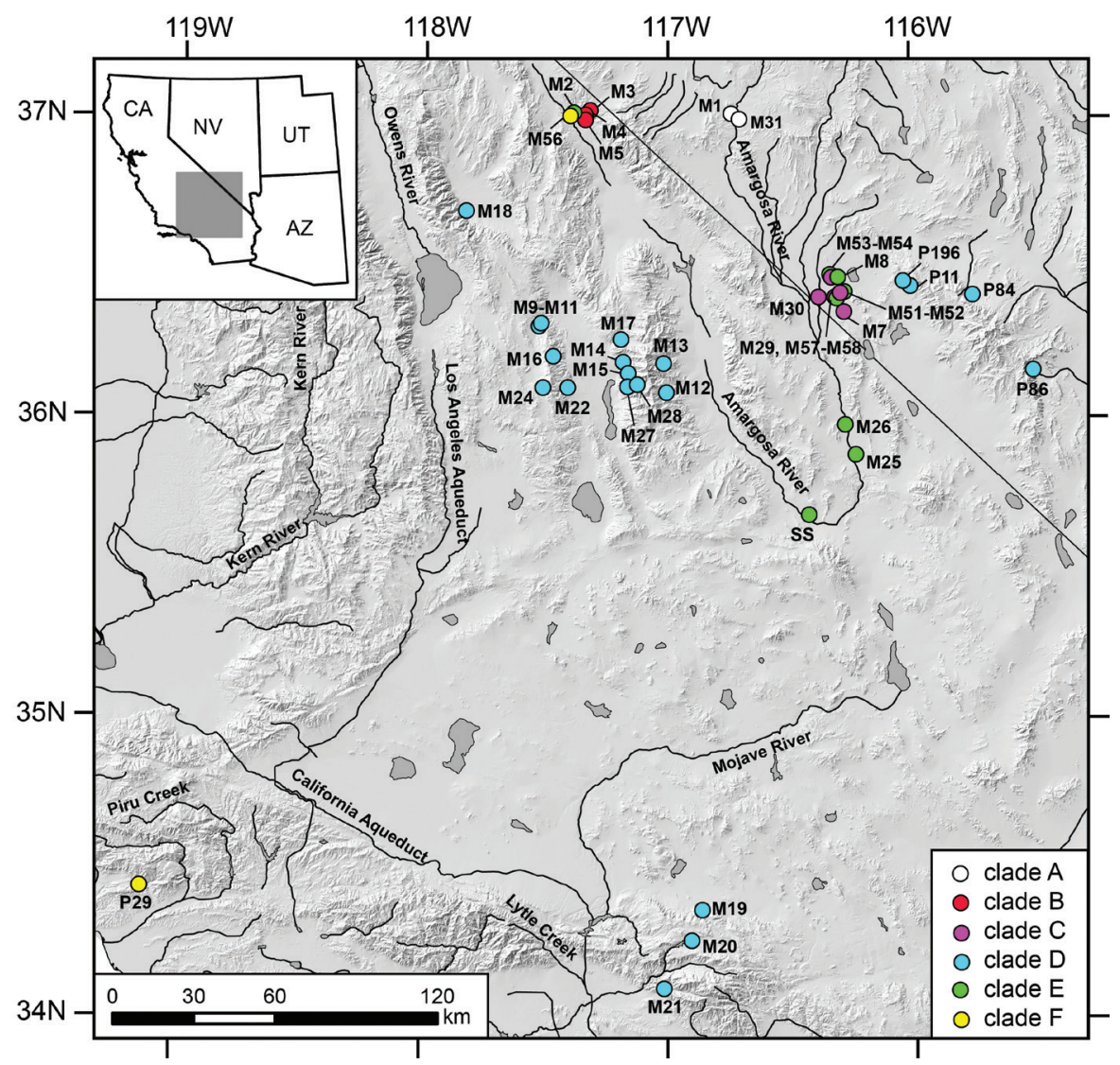

Figure 2. Map showing the distribution of mtDNA clades $\mathbf{A}-\mathbf{F}$ with color codes matching those in Fig. 1.

\section{Systematic descriptions}

\section{Family Hydrobiidae}

Subfamily Nymphophilinae

\section{Genus Pyrgulopsis Call \& Pilsbry, 1886}

\section{Pyrgulopsis licina Hershler, Liu \& Bradford, sp. n.}

http://zoobank.org/5F006641-38E0-4AA0-B3D2-AE78145B51CC http://species-id.net/wiki/Pyrgulopsis_licina

Figs 3, 4A-B

Pyrgulopsis micrococcus.-Hershler and Sada 1987 (in part).

[Pyrgulopsis micrococcus] clade C.-Liu et al. (2003).

Types. Holotype, USNM 850347 (a dry shell), spring south of Clay Pits, Ash Meadows, Nye County, Nevada, $36.40719^{\circ} \mathrm{N}, 116.37856^{\circ} \mathrm{W}, 11$ November 1985, R. Hershler and D. W. Sada. Paratypes, USNM 1204732 (from same lot). 
Referred material. NEVADA. Nye County: USNM 859186, USNM 903997, spring south of Clay Pits, USNM 850345, USNM 850346, USNM 850347, USNM 850348, USNM 859185, spring at Clay Pits, Ash Meadows $\left(36.41608^{\circ} \mathrm{N}, 116.37802^{\circ} \mathrm{W}\right)$, USNM 850343, USNM 850344, USNM 859184, spring north of Clay Pits, Ash Meadows $\left(36.41613^{\circ} \mathrm{N}, 116.37808^{\circ} \mathrm{W}\right)$, USNM 850334, Rogers Spring, Ash Meadows $\left(36.47931^{\circ} \mathrm{N}, 116.32622^{\circ} \mathrm{W}\right)$, USNM 850336, USNM 1122742, USNM 1122754, USNM 1197782, USNM 1204745, springs south of Rogers Spring, Ash Meadows $\left(36.47467^{\circ} \mathrm{N}, 116.32747^{\circ} \mathrm{W}\right)$, USNM 850349 , USNM 1197775 , spring east of Crystal Reservoir, Ash Meadows $\left(36.40790^{\circ} \mathrm{N}, 116.31297^{\circ} \mathrm{W}\right)$, USNM 850350, spring east of Crystal Reservoir, Ash Meadows (36.40742 N, $\left.116.31197^{\circ} \mathrm{W}\right)$, USNM 903982, spring east of Crystal Reservoir, Ash Meadows $\left(36.40836^{\circ} \mathrm{N}, 116.31042^{\circ} \mathrm{W}\right)$, USNM 1197780 , spring ca. $100 \mathrm{~m}$ north of Collins Ranch, Ash Meadows $\left(36.42038^{\circ} \mathrm{N}\right.$, $\left.116.29921^{\circ} \mathrm{W}\right)$, USNM 850352, USNM 850351, USNM 859188, USNM 859189, USNM 1122848, Frenchy Springs, Ash Meadows (36.36364 $\left.\mathrm{N}, 116.27432^{\circ} \mathrm{W}\right)$, USNM 850353, USNM 859190, USNM 894336, USNM 1122849, Last Chance Spring, Ash Meadows $\left(36.35700^{\circ} \mathrm{N}, 116.27400^{\circ} \mathrm{W}\right)$.

Diagnosis. A small congener (maximum shell height, $2.4 \mathrm{~mm}$ ) having a narrowconic shell. Distinguished from similar regional species by its strongly curved penial filament and absence of glands on the penis. Further differentiated from frequently sympatric $P$. sanchezi (described below) by its highly convex, deeply incised teleoconch whorls and ovate shell aperture.

Description. Shell (Fig. 3A-C) narrow-conic, whorls 3.75-4.50. Teleoconch whorls highly convex, sutures deeply impressed. Aperture ovate, parietal lip complete, narrowly adnate or slightly disjunct, umbilicus narrow. Outer lip thin, orthocline or prosocline. Sculpture of faint, irregular spiral striae.

Operculum (Fig. 3D-E) as for genus; edges of last 0.5 whorl frilled on outer side; muscle attachment margins variably thickened on inner side. Radula (Fig. $3 \mathrm{~F}-\mathrm{H})$ as for genus; dorsal edge of central teeth concave, lateral cusps four-six, basal cusp one. Lateral teeth having three-four cusps on both inner and outer sides. Inner marginal teeth with 20-25 cusps, outer marginal teeth with 24-31 cusps. Radula data are from USNM 850348.

Penis (Fig. 4A-B) medium-sized; filament medium length, narrow, weakly tapering, strongly curved (to outer side); lobe small, rectangular, horizontal or oblique; glands almost always absent (87/90 specimens), two specimens had a small, dot-like

Table I. Shell parameters for P. licina. Measurements are in $\mathrm{mm}$.

\begin{tabular}{|c|c|c|c|c|c|c|c|c|c|c|}
\hline & WH & SH & SW & HBW & WBW & AH & AW & SW/SH & $\begin{array}{c}\text { HBWI } \\
\text { SH }\end{array}$ & AH/SH \\
\hline \multicolumn{11}{|c|}{ Holotype, USNM 850347} \\
\hline & 4.25 & 1.94 & 1.33 & 1.42 & 1.09 & 0.78 & 0.76 & 0.69 & 0.73 & 0.44 \\
\hline \multicolumn{11}{|c|}{ USNM $1204732(\mathrm{n}=10)$} \\
\hline Mean & 4.00 & 2.00 & 1.33 & 1.45 & 1.15 & 0.83 & 0.76 & 0.67 & 0.73 & 0.42 \\
\hline S.D. & 0.12 & 0.12 & 0.06 & 0.08 & 0.06 & 0.05 & 0.03 & 0.03 & 0.02 & 0.02 \\
\hline Range & $3.75-4.25$ & $1.88-2.22$ & $1.28-1.44$ & $1.34-1.60$ & $1.09-1.27$ & $0.75-0.92$ & $0.72-0.83$ & $0.63-0.72$ & $0.70-0.76$ & $0.38-0.45$ \\
\hline
\end{tabular}




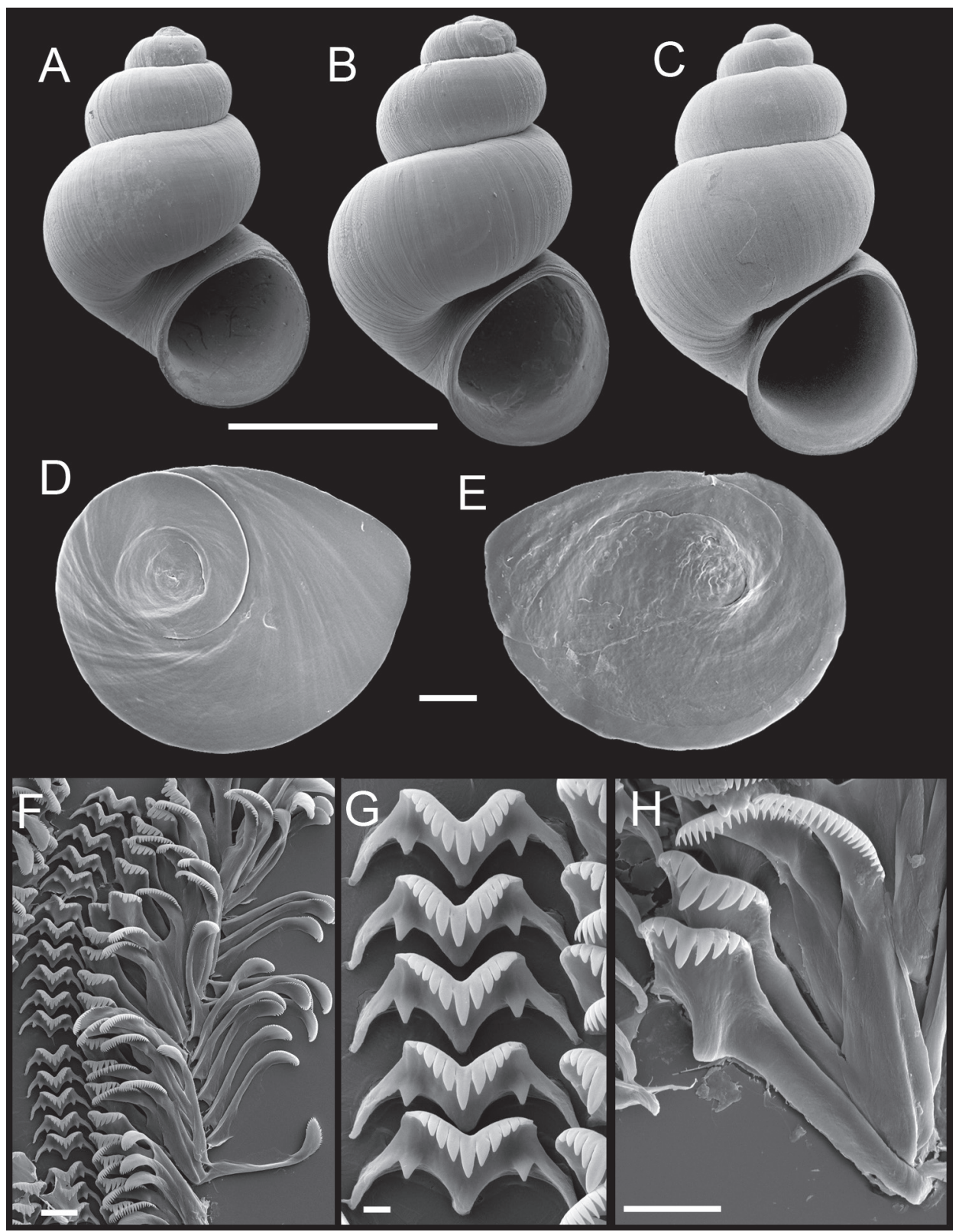

Figure 3. Shells, opercula and radula, P. licina sp. n. A Holotype, USNM 850347 B, C Shells, USNM 1204732, USNM 1188732 D, E Opercula (outer, inner sides), USNM 850348 F Portion of radular ribbon, USNM 850348 G Central teeth, USNM $850348 \mathbf{H}$ Lateral and inner marginal teeth, USNM 850348. Scale bars A-C 1.0 mm; D, E $100 \mu \mathrm{m}$; F, H $10 \mu \mathrm{m}$; G $2 \mu \mathrm{m}$.

gland along the distal edge of the lobe and one specimen had a glandular smear near the distal edge of the ventral surface of the lobe. Penial data are from USNM 850334, USNM 850348, USNM 850351. 

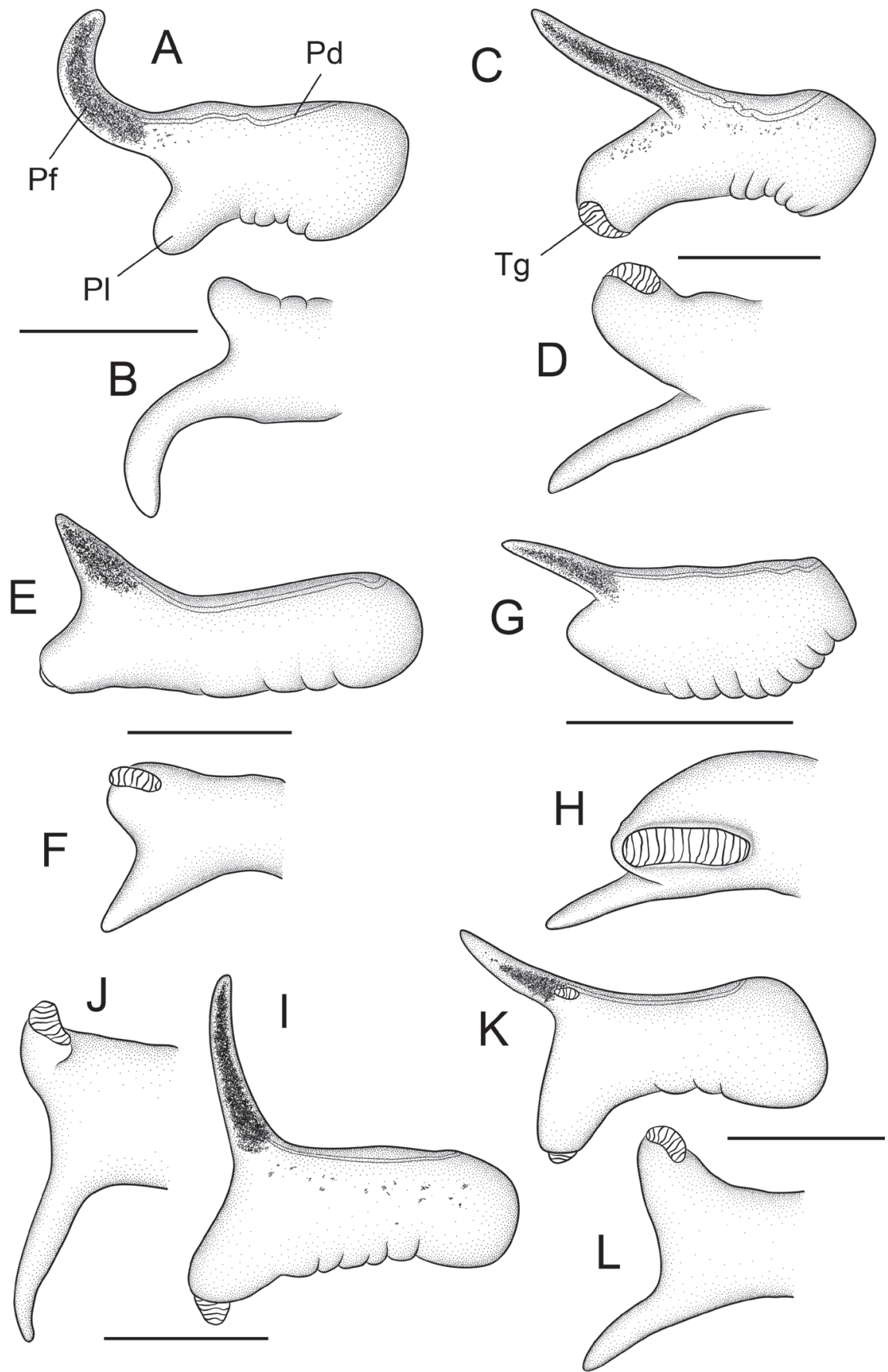

Figure 4. Penes (dorsal, ventral surfaces). A, B $P$. licina sp. n., USNM 850346 C, D $P$. perforata sp. n., BellMNH 20891 E, F P. sanchezi sp. n., USNM 883361 G, H P. micrococcus, BellMNH 20663 I, J $P$. turbatrix, USNM 860699 K, L $P$. turbatrix, USNM 883373. Scale bars A-C $250 \mu \mathrm{m}$; D-L $500 \mu \mathrm{m}$. Pd penial duct Pf penial filament $\mathbf{P l}$ penial lobe Tg terminal gland. 
Etymology. The epithet is an adjective derived from the New Latin licinus, meaning bent or turned upward, and refers to the distinctive shape of the penial filament in this species.

Distribution. Ash Meadows, Amargosa River basin (M7, M29, M30, M52, M54, M58, Fig. 2). The type locality is a broad spring brook that courses through a pit-like depression (Fig. 5A).

Remarks. The relationships of $P$. licina were not well resolved in the molecular phylogenetic analysis (Fig. 1). Haplotype variation within this clade was relatively small (Appendix II).

\section{Pyrgulopsis perforata Hershler, Liu \& Bradford, sp. n.}

http://zoobank.org/B246F89B-53AD-4476-8F47-DE21324ED20F

http://species-id.net/wiki/Pyrgulopsis_perforata

Figs 4C-D, 6

Pyrgulopsis micrococcus.-Hershler 1989 (in part).

[Pyrgulopsis micrococcus] clade B.-Liu et al. (2003).

Types. United States: Holotype, USNM 853507 (a dry shell), easternmost spring from Scotty's Castle along California Highway 72, Grapevine Canyon, Death Valley, Inyo County, California, $37.03233^{\circ} \mathrm{N}, 117.32333^{\circ} \mathrm{W}, 26$ February 1985, R. Hershler. Paratypes, USNM 1204734 (from same lot).

Referred material. CALIFORNIA. Inyo County: BellMNH 20891, USNM 857965, USNM 883371, USNM 883374, USNM 883375, USNM 883376, USNM 883377, USNM 894332, easternmost spring from Scotty's Castle along CA Hwy 72, Grapevine Canyon, Death Valley, USNM 883369, spring east of Scotty's Castle along CA Hwy 27, Grapevine Canyon, Death Valley (37.03259 $\left.{ }^{\circ} \mathrm{N}, 117.33118^{\circ} \mathrm{W}\right)$, USNM 883368, USNM 883379 , spring just east of Scotty's Castle, Grapevine Canyon, Death Valley $\left(37.03205^{\circ} \mathrm{N}\right.$, $\left.117.33715^{\circ} \mathrm{W}\right)$, BellMNH 20999, USNM 894333, spring ca. $0.8 \mathrm{~km}$ west of Scotty's Castle along CA Hwy 72 , Grapevine Canyon, Death Valley $\left(37.01400^{\circ} \mathrm{N}, 117.34867^{\circ} \mathrm{W}\right)$, USNM 894334, Surprise Springs, Death Valley $\left(36.99933^{\circ} \mathrm{N}, 117.34400^{\circ} \mathrm{W}\right)$.

Diagnosis. A small to medium-sized congener (maximum shell height, $2.6 \mathrm{~mm}$ ) having a broadly to ovate conic shell. Differentiated from similar regional species except $P$. micrococcus by its low-spired, broadly umbilicate shell. Differs from $P$. micrococcus in having a larger distal lobe and smaller gland on the penis.

Description. Shell (Fig. 6A-B) broadly to ovate conic, whorls 3.00-4.25. Teleoconch whorls medium convex, shouldered. Aperture ovate, parietal lip complete, narrowly adnate or slightly disjunct, last $0.25-0.5$ whorl rarely loosened behind aperture, umbilicus broad (Fig. 6C). Outer lip thin, orthocline or prosocline.

Operculum (Fig. 6D-E) as for genus; outer side smooth; inner side smooth or weakly thickened along portions of the muscle attachment margin. Radula (Fig. 6F$\mathrm{H}$ ) as for genus; dorsal edge concave, lateral cusps four-eight, basal cusp one. Lateral 

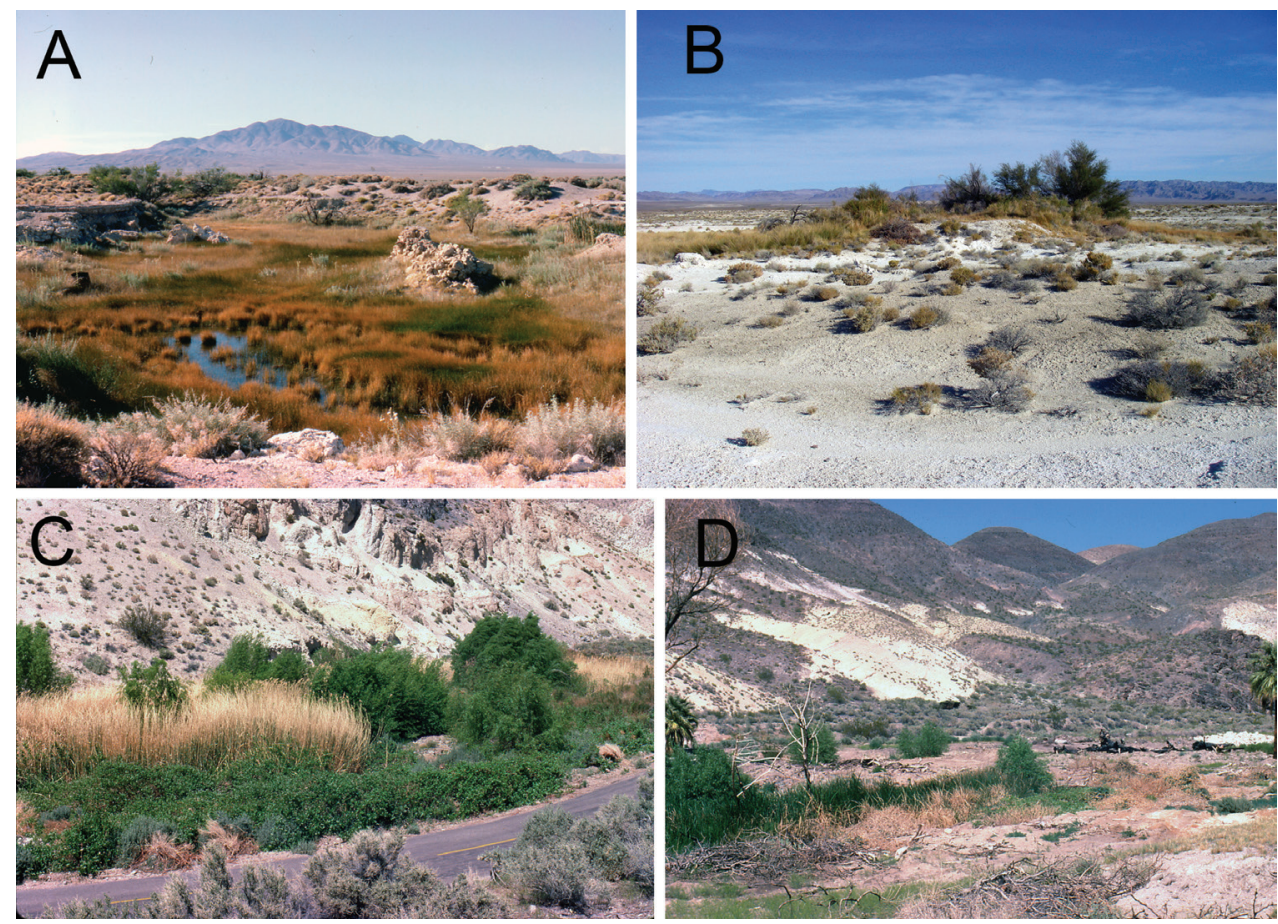

Figure 5. Photographs of habitats. A Spring south of Clay Pits, Ash Meadows, Nye County, Nevada, type locality of $P$. licina sp. n. (photograph taken on 7/VII/1986) B Purgatory Spring, Ash Meadows, Nye County, Nevada, type locality of $P$. sanchezi (15/XI/2011) C, D Uppermost spring east of Scotty's Castle, Death Valley, Inyo County, California, type locality of $P$. perforata sp. n. (18/IV/1980).

Table 2. Shell parameters for $P$. perforata. Measurements are in $\mathrm{mm}$.

\begin{tabular}{l|c|c|c|c|c|c|c|c|c|c}
\hline & WH & SH & SW & HBW & WBW & AH & AW & SW/SH & HBW/SH & AH/SH \\
\hline \multicolumn{1}{l}{ Holotype, USNM 853507} \\
\hline & 3.75 & 1.82 & 1.51 & 1.46 & 1.30 & 0.87 & 0.79 & 0.83 & 0.80 & 0.48 \\
\hline \multicolumn{2}{l}{ USNM $1204734(\mathrm{n}=10)$} \\
\hline Mean & 3.63 & 1.83 & 1.52 & 1.50 & 1.29 & 0.91 & 0.82 & 0.84 & 0.82 & 0.50 \\
\hline S.D. & 0.21 & 0.21 & 0.08 & 0.13 & 0.09 & 0.07 & 0.05 & 0.08 & 0.03 & 0.04 \\
\hline Range & $3.25-4.00$ & $1.51-2.09$ & $1.41-1.67$ & $1.31-1.68$ & $1.15-1.41$ & $0.83-1.03$ & $0.75-0.90$ & $0.74-0.98$ & $0.77-0.87$ & $0.45-0.55$ \\
\hline
\end{tabular}

teeth having two-four cusps on inner sides and three-six cusps on outer sides. Inner marginal teeth with 14-24 cusps, outer marginal teeth with 18-31 cusps. Radula data are from USNM 857965.

Penis (Fig. 4C-D) medium-sized; filament medium length, narrow, tapering, oblique; lobe medium-sized, rectangular, horizontal or slightly oblique; small (terminal) gland present on ventral edge of lobe (60/60 specimens), one specimen had an additional dot-like gland on the ventral surface of the lobe and one specimen had a similar glandular unit on the dorsal surface of the lobe. Penial data are from BellMNH 20891, USNM 883371. 


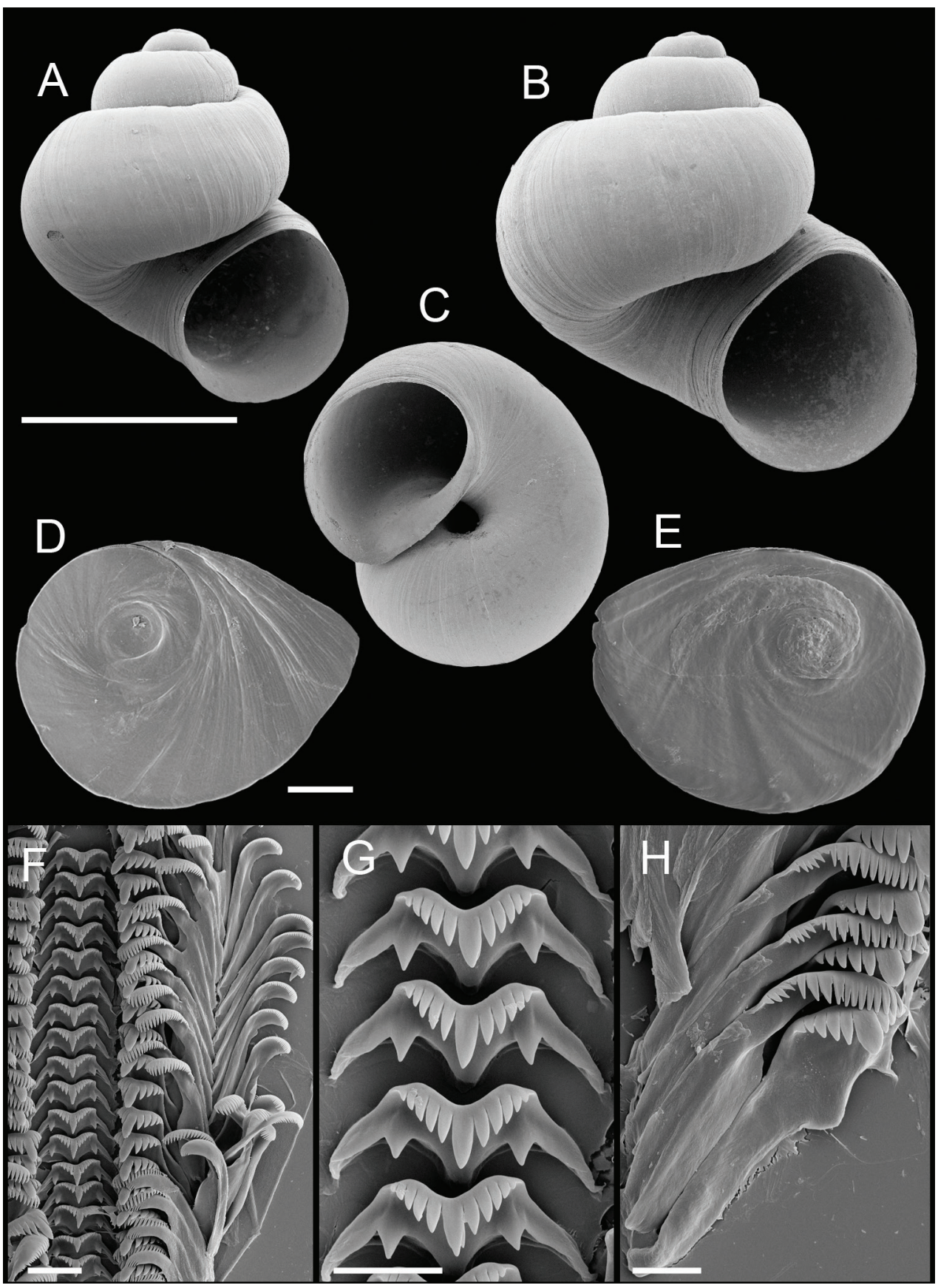

Figure 6. Shells, opercula and radula, $P$. perforata sp. n. A Holotype, USNM 853507 B, C Shells, USNM 1204734 D, E Opercula (outer, inner sides), USNM 857965 F Portion of radular ribbon, USNM 857965 G Central teeth, USNM 857965 H Lateral and inner marginal teeth, USNM 857965. Scale bars A-C 1.0 mm; D, E $200 \mu \mathrm{m} ; \mathbf{F} 20 \mu \mathrm{m}$; G, H $10 \mu \mathrm{m}$. 
Distribution. Lower portion of Grapevine Canyon, and Grapevine Mountains, lower Amargosa River basin (M3, M4, M5, Fig. 2). The type locality (Fig. 5C-D) is the uppermost of a small series of springs to the east of Scotty's Castle.

Etymology. An adjective derived from the New Latin, perforare, meaning to pierce, and referring to the broad umbilicate shells of this species.

Remarks. The relationships of $P$. perforata were not well resolved in the molecular phylogenetic analysis (Fig. 1). Haplotype variation within this clade was relatively small (Appendix II).

\section{Pyrgulopsis sanchezi Hershler, Liu \& Bradford, sp. n. http://zoobank.org/DA1A41D8-E257-466B-B28E-E500E89D16A9 http://species-id.net/wiki/Pyrgulopsis_sanchezi Figs 4E-F, 7 \\ Pyrgulopsis micrococcus.-Hershler and Sada 1987 (in part). \\ Pyrgulopsis micrococcus.-Hershler 1989 (in part). \\ [Pyrgulopsis micrococcus] clade E.-Liu et al. (2003).}

Types. United States: Holotype, USNM 850333 (a dry shell), Purgatory Spring, Ash Meadows, Nye County, Nevada, $36.47200^{\circ} \mathrm{N}, 116.31617^{\circ} \mathrm{W}, 26$ February 1985, R. Hershler and D.W. Sada. Paratypes, USNM 1204735 (from same lot).

Referred material. CALIFORNIA. Inyo County: USNM 853505, USNM 853506, USNM 854609, USNM 854610, Grapevine Springs, spring brook on travertine bench above Scotty's Ranch, Death Valley $\left(37.019210^{\circ} \mathrm{N}, 117.38649^{\circ} \mathrm{W}\right)$, USNM 857964, USNM 883372, USNM 1152507, Grapevine Springs, spring outflow at Scotty's Ranch, Death Valley $\left(37.01830^{\circ} \mathrm{N}, 117.38770^{\circ} \mathrm{W}\right)$, USNM 1197772 , Grapevine Springs, spring outflow below Scotty's Ranch, Death Valley $\left(37.01760^{\circ} \mathrm{N}\right.$, $\left.117.39420^{\circ} \mathrm{W}\right)$, USNM 853503, Grapevine Springs, northern-most spring complex, outflow below base of hill, Death Valley $\left(37.01970^{\circ} \mathrm{N}, 117.39288^{\circ} \mathrm{W}\right)$, USNM 894331, Grapevine Springs, third stream north of ranch, Death Valley $\left(37.01867^{\circ} \mathrm{N}\right.$, $\left.117.38900^{\circ} \mathrm{W}\right)$, BellMNH 21116, USNM 853501, USNM 857962, USNM 894335, USNM 1152506, Shoshone Spring, (35.98022 N, $\left.116.27308^{\circ} \mathrm{W}\right)$, USNM 853502, USNM 857963, USNM 873153, USNM 883366, USNM 894354, Tecopa Hot Springs, northern-most spring, $\left(35.88011^{\circ} \mathrm{N}, 116.22992^{\circ} \mathrm{W}\right)$, USNM 874035 , Spring brook north of Tecopa, $\left(35.85346^{\circ} \mathrm{N}, 116.22361^{\circ} \mathrm{W}\right)$. San Bernardino County: USNM 123904, USNM 883365, USNM 899902, USNM 1008345, USNM 1008725, USNM 1011485, USNM 1152503, Saratoga Springs, Death Valley $\left(35.68099^{\circ} \mathrm{N}\right.$, $\left.116.42245^{\circ} \mathrm{W}\right)$. NEVADA. Nye County: USNM 850339, USNM 859183, USNM 1122825 , Shaft Spring, Ash Meadows $\left(36.45109^{\circ} \mathrm{N}, 116.31552^{\circ} \mathrm{W}\right)$, USNM 850340, USNM 1122826, Chalk Spring, Ash Meadows ( $\left.36.44913^{\circ} \mathrm{N}, 116.31497^{\circ} \mathrm{W}\right)$, BellMNH 20664, School Spring, Ash Meadows (36.42741 $\left.\mathrm{N}, 116.30397^{\circ} \mathrm{W}\right)$, USNM 
1204746, Rogers Spring, Ash Meadows (36.47931 $\left.{ }^{\circ} \mathrm{N}, 116.32632^{\circ} \mathrm{W}\right)$, USNM 850335, USNM 859180, USNM 859181, USNM 204755, USNM 1122554, springs south of Rogers Spring, Ash Meadows (36.47467 $\left.\mathrm{N}, 116.32747^{\circ} \mathrm{W}\right)$, USNM 850337, USNM 850338, Five Springs, Ash Meadows ( $\left.36.46476^{\circ} \mathrm{N}, 116.32023^{\circ} \mathrm{W}\right)$, USNM 859182, USNM 1122821, spring south of Five Springs, Ash Meadows $\left(36.45109^{\circ} \mathrm{N}\right.$, $\left.116.31552^{\circ} \mathrm{W}\right)$, BellMNH 20666, BellMNH 20743, BellMNH 21149, USNM 850341, USNM 850342, USNM 859195, USNM 1204752, spring ca. $100 \mathrm{~m}$ north of Collins Ranch, Ash Meadows $\left(36.42038^{\circ} \mathrm{N}, 116.29921^{\circ} \mathrm{W}\right)$, BellMNH 20741, USNM 859179, USNM 883361, USNM 894337, USNM 1074313, USNM 1122759, USNM 1152498, Purgatory Spring, Ash Meadows, USNM 1204738, USNM 1197773, spring east of Crystal Reservoir, Ash Meadows $\left(36.40790^{\circ} \mathrm{N}\right.$, $\left.116.31297^{\circ} \mathrm{W}\right)$, USNM 859187, USNM 1204744, spring east of Crystal Reservoir, Ash Meadows $\left(36.40742^{\circ} \mathrm{N}, 116.31197^{\circ} \mathrm{W}\right)$.

Diagnosis. A small to medium-sized congener (maximum shell height, $2.9 \mathrm{~mm}$ ) having an ovate to narrow conic shell. Differentiated from similar regional species by its short, strongly tapering penial filament.

Description. Shell (Fig. 7A-D) ovate to narrow conic, whorls 3.5-4.75. Teleoconch whorls medium convex, sometimes strongly shouldered, last $0.25-0.50$ whorl sometimes slightly loosened. Aperture ovate, sometime strongly angled adapically, parietal lip complete, narrowly adnate or slightly disjunct, umbilicus usually narrow. Apertural lip sometimes rather thickened and/or slightly reflected, outer lip orthocline or prosocline.

Operculum (Fig. 7E-F) as for genus; outer side smooth or with last 0.5 whorl weakly frilled; inner side smooth or slightly thickened along a small portion of the muscle attachment margin. Radula (Fig. 7G-I) as for genus; dorsal edge concave, lateral cusps three-six, basal cusp one. Lateral teeth having one-four cusps on inner sides and two-six cusps on outer sides. Inner marginal teeth with 10-26 cusps, outer marginal teeth with 12-33 cusps. Radula data are from BellMNH 21116, USNM 857963, USNM 883361, USNM 883365, USNM 883372.

Penis (Fig. 4E-F) medium-sized; filament short, broad, strongly tapering, oblique; lobe short, rectangular, horizontal or slightly oblique; small (terminal) ovate gland almost always present on ventral surface of lobe ( $92 / 93$ specimens), gland usually positioned horizontally, rarely borne on a raised swelling (one specimen), one specimen had a second, dot-like gland on the ventral surface of the lobe. Penial data are from BellMNH 2116, USNM 857963, USNM 857964, USNM 883361, USNM 883666.

Table 3. Shell parameters for $P$. sanchezi. Measurements are in $\mathrm{mm}$.

\begin{tabular}{|c|c|c|c|c|c|c|c|c|c|c|}
\hline & WH & SH & SW & HBW & WBW & AH & AW & SW/SH & HBW/SH & $\mathrm{AH} / \mathrm{SH}$ \\
\hline \multicolumn{11}{|c|}{ Holotype, USNM 850333} \\
\hline & 4.25 & 2.50 & 1.73 & 1.95 & 1.40 & 1.10 & 1.07 & 0.69 & 0.78 & 0.44 \\
\hline \multicolumn{11}{|c|}{ USNM $1204735(\mathrm{n}=10)$} \\
\hline Mean & 4.33 & 2.43 & 1.66 & 1.82 & 1.33 & 1.06 & 0.99 & 0.68 & 0.75 & 0.43 \\
\hline S.D. & 0.21 & 0.14 & 0.10 & 0.10 & 0.06 & 0.06 & 0.07 & 0.04 & 0.02 & 0.02 \\
\hline Range & $4.00-4.75$ & $2.22-2.70$ & $1.46-1.81$ & $1.68-1.94$ & $1.22-1.44$ & $0.96-1.16$ & $0.90-1.08$ & $0.62-0.73$ & $0.70-0.78$ & $0.40-0.46$ \\
\hline
\end{tabular}




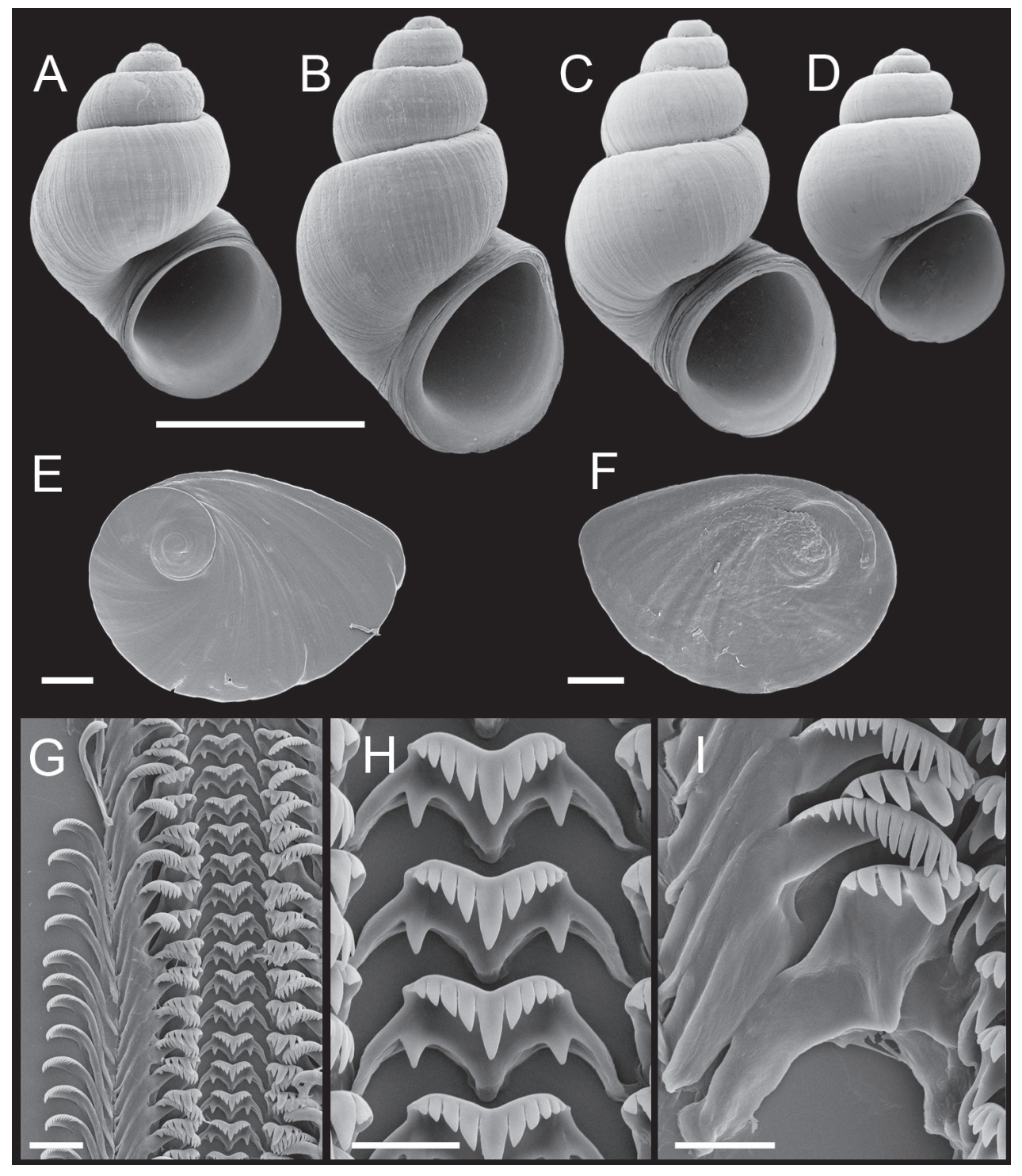

Figure 7. Shells, opercula and radula, P. sanchezi sp. n. A Holotype, USNM 850333 B-D Shells, USNM 853505, USNM 1204755, USNM 853501 E, F Opercula (outer, inner sides), USNM 883361 G Portion of radular ribbon, USNM 883361 H Central teeth, USNM 883361 I Lateral and inner marginal teeth, USNM 883361. Scale bars A-D 1.0 mm; E, F $100 \mu \mathrm{m}$; G $20 \mu \mathrm{m}$; H, I $10 \mu \mathrm{m}$.

Distribution. Distributed in five separate groundwater discharge areas of the Amargosa River basin: Grapevine Springs (M2), Ash Meadows (M8, M51, M53, M57), Tecopa (M25), Shoshone (M26), Saratoga Spring (SS) (Fig. 2). The type locality (Fig. 5B) is a flowing well that was drilled into a small spring mound (Dudley and Larson 1976).

Etymology. This species is named for Peter G. Sanchez, who spearheaded early efforts to protect and conserve regional springsnails and their associated aquatic 
habitats while serving as a Resource Management Specialist in the Death Valley National Monument (now National Park) and Chair of the Desert Fishes Council (1978-1980).

Remarks. Pyrgulopsis sanchezi was resolved as sister to $P$. arizonae (Gila River basin, Arizona) in the Bayesian analysis (Fig. 1). The five geographically separated groups of $P$. sanchezi populations are genetically differentiated-e.g., mean genetic distance is $1.5+/-0.3 \%$ (ranging from $1.3-2.3 \%$ ) for COI and $2.1+/-0.6 \%$ (ranging from $1.8-$ $3.2 \%$ ) for NDI, however we have not found consistent morphological differences to support their recognition as distinct species.

\section{Pyrgulopsis micrococcus (Pilsbry, 1893)}

http://species-id.net/wiki/Pyrgulopsis_micrococcus

Figs 4G-H, 8

Amnicola micrococcus Pilsbry in Stearns 1893: 277, fig. 1 (small spring in Oasis Valley

Nevada; also from Death Valley). Baker 1964: 174 (lectotype designation).

Fontelicella (Microamnicola) micrococcus.-Gregg and Taylor 1965: 109 (comb. n.).

Pyrgulopsis micrococcus.-Hershler and Thompson 1987: 29 (new combination). Hershler and

Sada 1987: 788-791 (in part). Hershler 1989: 182-187 (in part). Hershler 1998: 15. [Pyrgulopsis micrococcus] clade A.-Liu et al. (2003).

Types. Lectotype, ANSP 67279; paralectotypes, ANSP 368399, USNM 123622 (from same lot).

Other material examined. NEVADA. Nye County: USNM 847246, springs at Springdale $\left(37.03049^{\circ} \mathrm{N}, 116.75117^{\circ} \mathrm{W}\right)$, BellMNH 20674, spring east of Springdale $\left(37.03858^{\circ} \mathrm{N}, 116.71730^{\circ} \mathrm{W}\right)$, BellMNH 20671, BellMNH 20672, BellMNH 20673, BellMNH 20739, USNM 850297, USNM 857961, USNM 874778, USNM 894330, USNM 905091, USNM 1002348, USNM 1004184, USNM 1004185, USNM 1068649, USNM 1068650, USNM 1068794, spring at Fleur de Lis Ranch, ca. $0.8 \mathrm{~km}$ south of Springdale $\left(37.01700^{\circ} \mathrm{N}, 116.73300^{\circ} \mathrm{W}\right)$, BellMNH 20670, USNM 874771, USNM 1002349, USNM 1004182, USNM 1004183, USNM 1146338, Goss Springs $\left(36.99906^{\circ} \mathrm{N}, 116.70725^{\circ} \mathrm{W}\right)$, BellMNH 20669, BellMNH 20774, Ute Springs $\left(36.95729^{\circ} \mathrm{N}, 116.71648^{\circ} \mathrm{W}\right)$, BellMNH 20667, BellMNH 20740, USNM 874758, Revert Springs $\left(36.91795^{\circ} \mathrm{N}, 116.74397^{\circ} \mathrm{W}\right)$.

Revised diagnosis. A medium-sized congener (maximum shell height, $4.4 \mathrm{~mm}$ ) having a broadly to elongate conic shell. Differentiated from similar regional species by the large size of the gland on the ventral surface of the penis.

Description. Shell (Fig. 8A-D) broadly to narrow-conic, whorls 3.50-5.0. Teleoconch whorls weakly to strongly convex, sutures impressed. Aperture ovate, parietal lip complete, usually disjunct, last $0.25-0.5$ whorl often loosened behind aperture, umbilicus small. Outer lip usually thin, orthocline. Sculpture of faint, irregular spiral striae. 


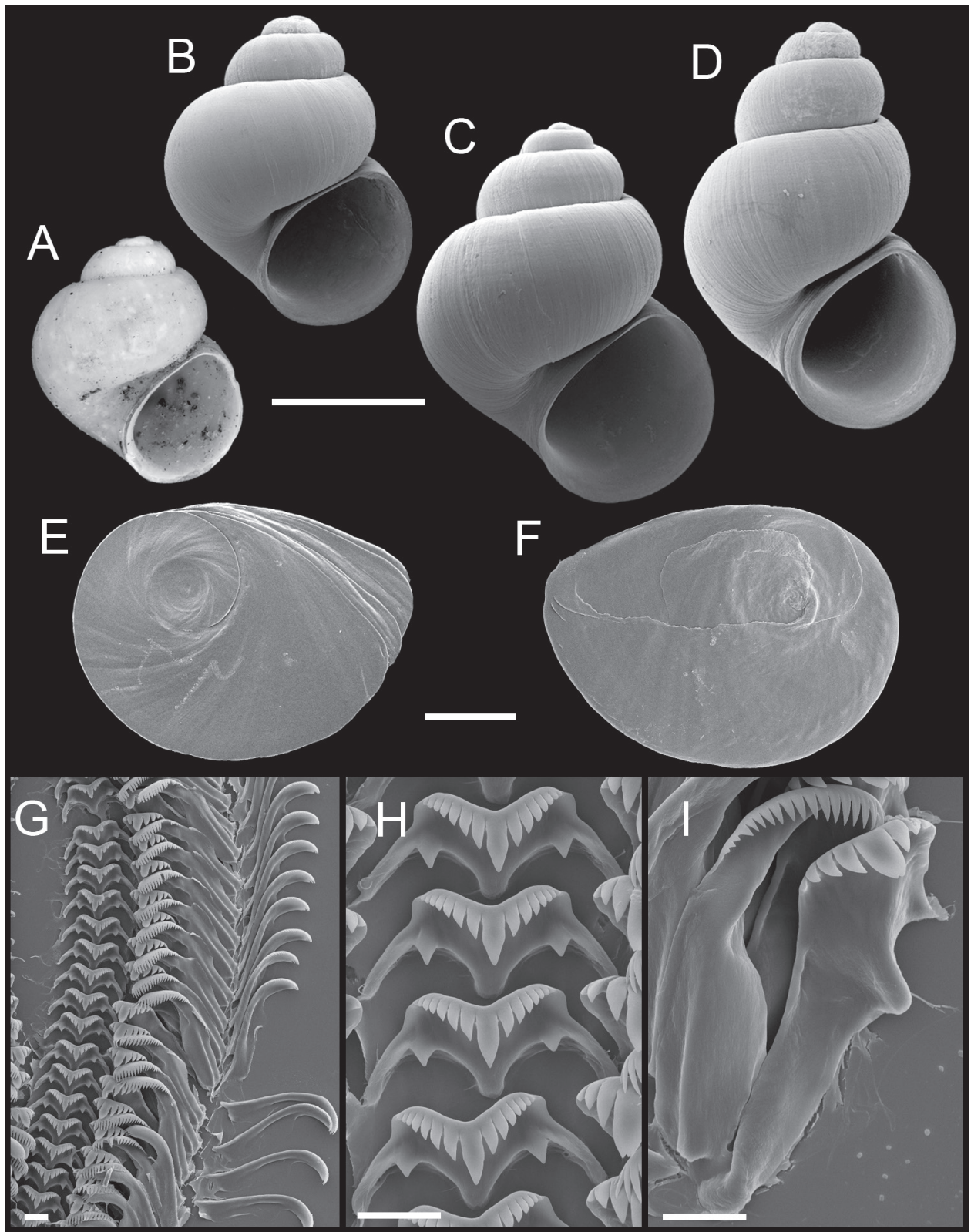

Figure 8. Shells, opercula and radula, $P$. micrococcus. A Lectotype, ANSP 67279a B-D Shells, USNM 1004185, USNM 905091, USNM 1004183 E, F Opercula (outer, inner sides), USNM 847246 G Portion of radular ribbon, USNM $847246 \mathbf{H}$ Central teeth, USNM 847246 I Lateral and inner marginal teeth, USNM 847246. Scale bars A-D 1.0 mm; E, F $250 \mu \mathrm{m}$; G-I $10 \mu \mathrm{m}$.

Operculum (Fig. 8E-F) as for genus; edges of last 0.5 whorl frilled on outer side; muscle attachment margins thickened on inner side. Radula (Fig. 8G-I) as for genus; dorsal edge of central radular teeth concave, lateral cusps five-eight, basal cusp 
one. Lateral teeth having three-four cusps on inner sides and four-five cusps on outer sides. Inner marginal teeth with 18-23 cusps, outer marginal teeth with 21-29 cusps. Radula data are from USNM 847246.

Penis (Fig. 4G-H) medium-sized; filament short, narrow, tapering, slightly oblique; lobe small, tapering, horizontal; a large (terminal) gland (borne on a raised swelling) present on ventral surface of penis, extending from near mid-length almost to tip of lobe (90/90 specimens), one-two additional small glands sometimes present on ventral surface of lobe (8 specimens), one specimen had a glandular dot on the dorsal surface near the base of the filament. Penial data are from BellMNH 20663, BellMNH 20669, BellMNH 20744.

Distribution. Several groups of springs in Oasis Valley, upper Amargosa River basin (M1, M31, Fig. 2).

Remarks. Pilsbry (in Stearns 1893; also see Stearns 1901) listed a single "type" lot for P. micrococcus, [USNM] 123622, which is composed of six dry shells. Baker (1964) subsequently designated ANSP 67279a as the "type" without explaining his rationale for this action. ANSP 67279a (Fig. 8A) closely conforms to Pilsbry's description and figure and is also very similar to the USNM type material (Hershler and Sada 1987, fig. 8a). The labels associated with ANSP 67279a indicate that it was part of the original collection of $P$. micrococcus (made by C. Hart Merriam) and this lot was almost certainly known to Pilsbry, who was the curator of mollusks at the Academy of Natural Sciences during the time period when his description was prepared and published. Based on this evidence we conclude that ANSP 67279a is part of the type series and thus Baker's subsequent lectotype designation is valid.

Pyrgulopsis micrococcus was resolved in the Bayesian tree as sister to an undescribed species from the Amargosa Canyon, south of Tecopa (Fig. 1). Specimens assigned to $P$. micrococcus vary somewhat in size and shell shape, but are closely similar both genetically (Liu et al., 2003) and in penial morphology.

\section{Pyrgulopsis turbatrix Hershler, 1998}

http://species-id.net/wiki/Pyrgulopsis_turbatrix

Figs 4I-L, 9

Pyrgulopsis turbatrix Hershler, 1998: 50, figs. 6K, 18G-J, 30D-F (Horseshutem Springs, Pahrump Valley, Nye County, Nevada).

Pyrgulopsis micrococcus.-Hershler 1989 (in part).

Pyrgulopsis micrococcus.-Hershler and Pratt 1990 (in part).

[Pyrgulopsis micrococcus] clade D.-Liu et al. (2003).

Types. Holotype, USNM 883978; paratypes, USNM 860699 (from same lot).

Other material examined. CALIFORNIA. Inyo County: USNM 853508, USNM 883373, Hanaupah Spring, Hanaupah Canyon, Death Valley $\left(36.18684^{\circ} \mathrm{N}\right.$, $\left.117.02537^{\circ} \mathrm{W}\right)$, USNM 853512, spring above Darwin Falls, Panamint Valley 


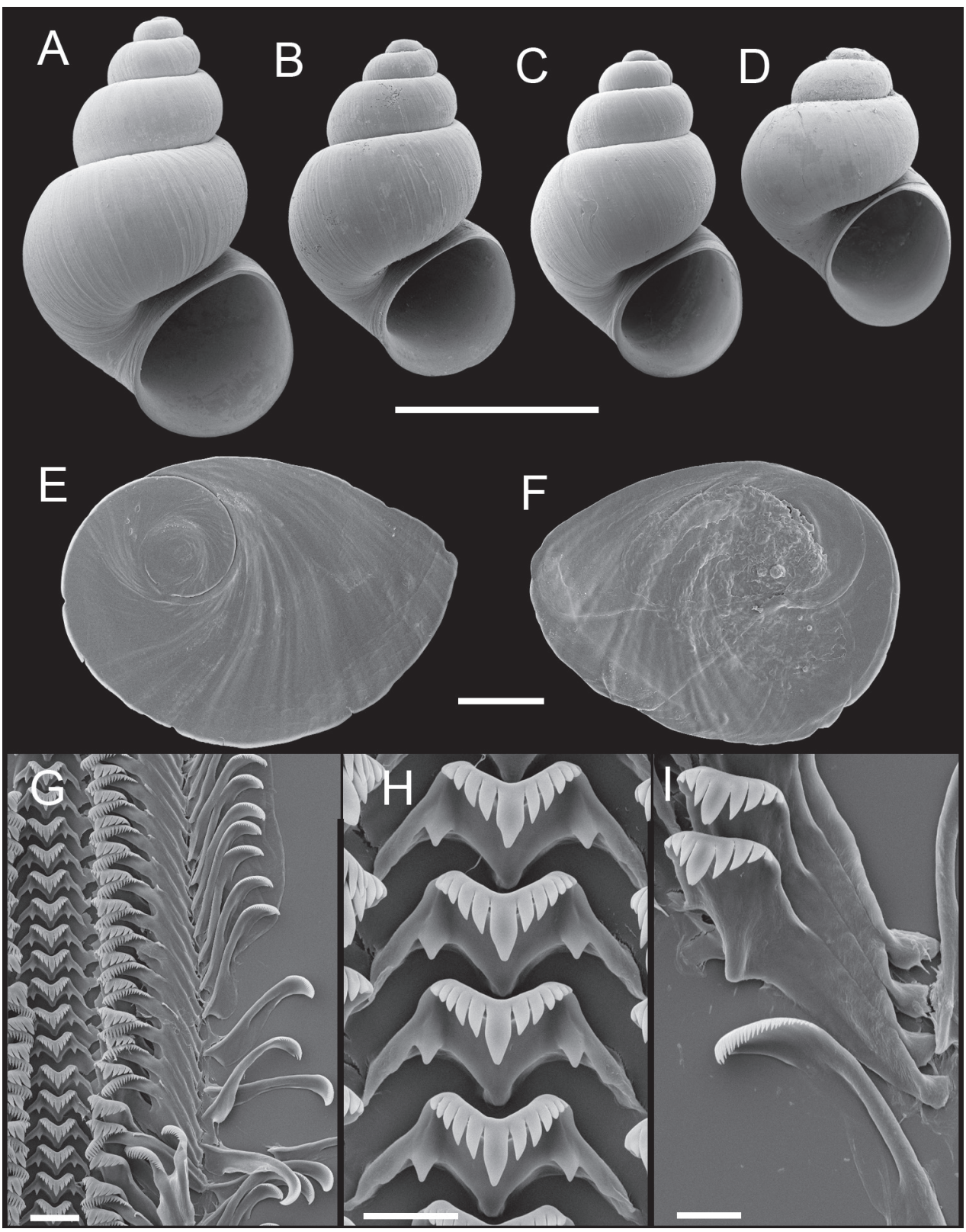

Figure 9. Shells, opercula and radula, $P$. turbatrix. A Paratype, USNM 860699. B-D Shells, SBMNH uncat., USNM 853508, USNM 853510 E, F Opercula (outer, inner sides), USNM 860699 G Portion of radular ribbon, USNM 860699 H Central teeth, USNM 860699 I Lateral and outer marginal teeth, USNM 860699. Scale bars A-D 1.0 mm; E, F $250 \mu \mathrm{m}$; G $20 \mu \mathrm{m}$; H, I $10 \mu \mathrm{m}$.

$\left(36.31783^{\circ} \mathrm{N}, 115.52700^{\circ} \mathrm{W}\right)$, USNM 857969, stream below Darwin Falls, Panamint Valley $\left(36.32033^{\circ} \mathrm{N}, 117.51917^{\circ} \mathrm{W}\right)$, USNM 857968, Saline Marsh, Saline Valley $\left(36.69350^{\circ} \mathrm{N}, 117.83033^{\circ} \mathrm{W}\right)$. San Bernardino County: SBMNH uncat., roadside 
spring between north shore highway and Big Bear Lake at point $1.2 \mathrm{~km}$ east of road which crosses lake, Southern California coastal drainage $\left(34.26424^{\circ} \mathrm{N}, 116.87497^{\circ} \mathrm{W}\right)$, USNM 860450, spring southwest of Big Bear Ranger Station, southern California coastal drainage $\left(34.26281^{\circ} \mathrm{N}, 116.90185^{\circ} \mathrm{W}\right)$. NEVADA. Clark County: USNM 883551, Willow Spring, Indian Springs Valley $\left(36.41700^{\circ} \mathrm{N}, 115.76419^{\circ} \mathrm{W}\right)$, USNM 883981 , La Madre Spring, Las Vegas Valley $\left(36.18381^{\circ} \mathrm{N}, 115.50638^{\circ} \mathrm{W}\right)$, USNM 1002785 , Harris Spring, Las Vegas Valley $\left(36.24071^{\circ} \mathrm{N}, 115.54351^{\circ} \mathrm{W}\right)$. Nye County: USNM 854967, Wood Canyon Spring, Pahrump Valley $\left(36.39924^{\circ} \mathrm{N}, 115.93258^{\circ} \mathrm{W}\right)$.

Revised diagnosis. A medium-sized congener (maximum shell height, $3.7 \mathrm{~mm}$ ) having an ovate to narrow-conic shell. Differentiated from similar regional species by the combination of its relatively large, narrow shell; elongate penial filament; and small size of the terminal gland on penis.

Description. Shell (Fig. 9A-D) ovate to narrow conic, rarely broadly conic, whorls 4.25-5.25. Teleoconch whorls strongly convex, shouldered. Aperture ovate, parietal lip complete, usually slightly disjunct, last 0.25 whorl sometimes loosened behind body whorl, umbilicus narrow. Outer lip thin, prosocline.

Operculum (Fig. 9E-F) as for genus; edges of last 0.5 whorl frilled on outer side; muscle attachment margins slightly thickened on inner side. Radula (Fig. 9G-I) as for genus; dorsal edge of central teeth moderately to highly concave, lateral cusps threeseven, basal cusps one-two. Lateral teeth having two-six cusps on inner sides and three-six cusps on outer sides. Inner marginal teeth with 14-31 cusps, outer marginal teeth with 15-33 cusps. Radula data are from USNM 857968, USNM 860450, USNM 860699, USNM 883373.

Penis (Fig. 4I-L) medium-sized; filament long, narrow, tapering, oblique; lobe medium-sized, tapering, slightly oblique; ventral surface of lobe having a small (terminal) gland (199/200 specimens) and rarely one or two additional glandular dots (3 specimens), dorsal surface sometimes having a small (penial) gland at base of filament (24/200 specimens) and rarely having an additional glandular dot (one specimen). Penial data are from USNM 854967, USNM 857969, USNM 860450, USNM 860699, USNM 883373, USNM 883981, USNM 1002785.

Distribution. Spring Mountains region (Frenchman Flat; Indian Springs, Las Vegas, Pahrump Valleys [P. turbatrix]), San Bernardino Mountains (Mojave, Southern California Coastal drainages [M19, M20, M21]), central Death Valley region (Amargosa River drainage, Panamint and Saline Valleys [M9-M22, M24, M27, M28]). The populations from the latter two areas were previously assigned to $P$. micrococcus.

Remarks. The penial gland was not observed in $>25 \%$ of the males in any of the seven samples that we studied and consequently has been removed from the diagnosis. The three geographically separated subunits of $P$. turbatrix are somewhat diverged genetically-e.g., mean sequence divergence is $0.9+/-0.2 \%$ (ranging from $1.1-1.5 \%$ ) for COI and $0.9+/-0.2 \%$ (ranging from $1.1-1.3 \%$ ) for NDI, but we have not found any consistent morphological differences among them. 


\section{Discussion}

The three novelties described herein increase the number of Pyrgulopsis species in the Death Valley region to 17 (Hershler and Sada 2002) and add to the large body of evidence supporting recognition of this desert area as one of the most significant hotspots of rarity and richness in the United States (Chaplin et al. 2000). Our revision of $P$. micrococcus obviously is not yet complete as we have not treated clade $\mathrm{F}$, which differs from the other lineages of $P$. micrococcus (in the broad sense) by $4.3-12.6 \%$ for COI and $7.4-13.3 \%$ for NDI (Appendix II). The two populations in clade $\mathrm{F}$ differ by only $0.8+/-0.3 \%$ for COI and $1.2+/-0.5 \%$ for NDI, suggesting that they may be conspecific. Additional studies are needed to clarify the taxonomy of this clade and to evaluate the biogeographic cause of its broadly disjunct distribution.

All of the new species described herein are endemic to the Amargosa River basin. Pyrgulopsis perforata and P. licina are distributed entirely within the confines of Death Valley National Park and Ash Meadows National Wildlife Refuge, respectively, and consequently are being afforded some measure of protection. The $P$. licina populations are also being monitored by The Nature Conservancy as part of their Nevada Springs Conservation Plan (Abele 2011). Three of the five genetically differentiated lineages of $P$. sanchezi are distributed in the land management areas mentioned above. The Tecopa lineage (M25) is distributed on private and public water resources lands and is being monitored as part of the Bureau of Land Management's Amargosa River Area of Environmental Concern Implementation Plan (BLM 2006). The Shoshone lineage (M26) lives in a spring on private land that has a long history of disturbance which includes diversion of most of its flow and extensive modification of its outflow channel (USFWS 1984, Castleberry et al. 1990). This population appears to be restricted at present to a small area of leakage from a spring box (RH, HPL, CB, personal observation 15.XI.2011) and some measures will need to be taken to ensure its long term persistence. Our finding that P. micrococcus is restricted to its type locality area (Oasis Valley) suggests a need for additional conservation-related activities. The known populations of this species are in disturbed springs (per Maciolek 1983) on private land and some protection is needed to ensure their long term persistence. Surveys are also needed to evaluate the current status of several populations of this species that have not been sampled for several decades (e.g., that in Ute Springs) and to determine whether there may be previously unrecorded populations in Oasis Valley, which contains a large area of groundwater discharge (Reiner et al. 2002). Pyrgulopsis micrococcus (as currently constituted) was a candidate for addition to the Federal List of Endangered and Threatened Wildlife and Plants (USFWS 1976) prior to its removal from this list in 1994 (USFWS 1994). 


\section{Acknowledgements}

We thank Paul Callomon and Amanda Lawless (ANSP), Andrew Simons and Jonathan Slaght (BellMNH), and Eric Hochberg and Paul Scott (SBMNH) for loans of specimens under their care. We thank Kevin P. Wilson (Death Valley National Park) and Sharon McKelvey (Ash Meadows National Wildlife Refuge) for provision of collection permits and logistical assistance in the field. We also thank Don Sada and Rob Robinson for collecting specimens that were used in this study. Yolanda Villacampa measured shells and prepared scanning electron micrographs, and Karolyn Darrow photographed the lectotype of $P$. micrococcus and inked the anatomical drawings.

\section{References}

Abele SL (2011) Nevada Springs Conservation Plan. Springs Conservation Plan Working Group. The Nature Conservancy, Reno, 61 pp. http://heritage.nv.gov/sites/default/files/ library/springcons_highres.pdf [accessed 17 June 2013]

Baker HB (1964) Type land snails in the Academy of Natural Sciences of Philadelphia Part III. Limnophile and thalassophile Pulmonata. Part IV. Land and freshwater Prosobranchia. Proceedings of the Academy of Natural Sciences of Philadelphia 116: 149-193.

BLM (Bureau of Land Management) (2006) [Draft] Amargosa River Area of Environmental Concern Implementation Plan. Barstow Field Office, CA, 285 pp. http://www.blm.gov/ ca/pdfs/barstow_pdfs/amargosa_ea/Complete.pdf [accessed 17 June 2013]

Bucklin A (1992) Use of formalin-preserved samples for molecular analysis. Newsletter of Crustacean Molecular Techniques 2: 3.

Castleberry DT, Williams JE, Sato GM, Hopkins TE, Brasher AM, Parker MS (1990) Status and management of Shoshone pupfish, Cyprinodon nevadensis shoshone (Cyprinodontidae), at Shoshone Spring, Inyo County, California. Bulletin of the South California Academy of Science 89: 19-25.

Chaplin SJ, Gerrard RA, Watson HM, Master LL, Flack SR (2000) The geography of imperilment: targeting conservation toward critical biodiversity areas. In: Stein BA, Kutner LS, Adams JS (Eds) Precious Heritage: the Status of Biodiversity in the United States. Oxford University Press, Oxford, 159-199.

Dudley WW Jr, Larson JD (1976) Effect of irrigation pumping on Desert pupfish habitats in Ash Meadows, Nye County, Nevada. United States Geological Survey Professional Paper 927: $1-52$.

Farris JS, Kallersjo M, Kluge AG, Bult C (1994) Testing significance of congruence. Cladistics 10: 315-319. doi: 10.1111/j.1096-0031.1994.tb00181.x

Folmer O, Black M, Hoeh W, Lutz R, Vrijenhoek R (1994) DNA primers for amplification of mitochondrial cytochrome $c$ oxidase subunit I from diverse metazoan invertebrates. Molecular Marine Biology and Biotechnology 3: 294-299. 
Gregg WO, Taylor DW (1965) Fontelicella (Prosobranchia: Hydrobiidae), a new genus of west American freshwater snails. Malacologia 3: 103-110.

Hershler R (1989) Springsnails (Gastropoda: Hydrobiidae) of Owens and Amargosa River (exclusive of Ash Meadows) drainages, Death Valley system, California-Nevada. Proceedings of the Biological Society of Washington 102: 176-248.

Hershler R (1994) A review of the North American freshwater snail genus Pyrgulopsis. Smithsonian Contributions to Zoology 554: 1-115. doi: 10.5479/si.00810282.554

Hershler R (1998) A systematic review of the hydrobiid snails (Gastropoda: Rissooidea) of the Great Basin, western United States. Part I. Genus Pyrgulopsis. The Veliger 41: 1-132.

Hershler R, Frest TJ, Liu H-P, Johannes EJ (2003) Rissooidean snails from the Pit River basin, California. Veliger 46: 275-304.

Hershler R, Liu H-P (2004) Taxonomic reappraisal of species assigned to the North American freshwater gastropod subgenus Natricola (Rissooidea: Hydrobiidae). Veliger 47: 66-81.

Hershler R, Liu H-P, Frest TJ, Johannes EJ (2007) Extensive diversification of pebblesnails (Lithoglyphidae: Fluminicola) in the upper Sacramento River basin, northwestern United States. Zoological Journal of the Linnean Society of London 149: 371-422.

Hershler R, Liu H-P (2008) Ancient vicariance and recent dispersal of springsnails (Hydrobiidae: Pyrgulopsis) in the Death Valley system, California-Nevada. In: Reheis MC, Hershler R, Miller DM (Eds) Late Cenozoic Drainage History of the Southwestern Great Basin and Lower Colorado River Region: Geologic and Biotic Perspectives. Geological Society of America Special Paper 439: 91-101. doi: 10.1130/2008.2439(04)

Hershler R, Liu H-P (2009) New species and records of Pyrgulopsis (Gastropoda: Hydrobiidae) from the Snake River basin, southeastern Oregon: further delineation of a highly imperiled fauna. Zootaxa 2006: 1-22.

Hershler R, Liu H-P (2012) A new species of springsnail (Pyrgulopsis) from the Owyhee River basin, Nevada. Western North American Naturalist 72: 21-31. doi: 10.3398/064.072.0103

Hershler R, Pratt WL (1990) A new Pyrgulopsis (Gastropoda: Hydrobiidae) from southeastern California, with a model for historical development of the Death Valley hydrographic system. Proceedings of the Biological Society of Washington 103: 279-299.

Hershler R, Sada DW (1987) Springsnails (Gastropoda: Hydrobiidae) of Ash Meadows, Amargosa basin, California-Nevada. Proceedings of the Biological Society of Washington 100: 776-843.

Hershler R, Sada DW (2002) Biogeography of Great Basin springsnails of the genus Pyrgulopsis. In: Hershler R, Madsen DB, Currey DR (Eds) Great Basin Aquatic Systems History. Smithsonian Contributions to the Earth Sciences 33, 255-276.

Hershler R, Thompson FG (1987) North American Hydrobiidae (Gastropoda: Rissoacea): redescription and systematic relationships of Tryonia Stimpson, 1865 and Pyrgulopsis Call and Pilsbry, 1886. Nautilus 101: 25-32.

Huelsenbeck JP, Ronquist F (2001) MRBAYES: Bayesian inference of phylogeny. Bioinformatics 17: 754-755. doi: 10.1093/bioinformatics/17.8.754

Hurt CR (2004) Genetic divergence, population structure and historical demography of rare springsnails (Pyrgulopsis) in the lower Colorado River basin. Molecular Ecology 13: 1173-1187. doi: 10.1111/j.1365-294X.2004.02121.x 
Liu H-P, Hershler R, Clift K (2003) Mitochondrial DNA sequences reveal extensive cryptic diversity within a western American springsnail. Molecular Ecology 12: 2771-2782. doi: 10.1046/j.1365-294X.2003.01949.x

Liu H-P, Hershler R (2005) Molecular systematics and radiation of western North American nymphophiline gastropods. Molecular Phylogenetics and Evolution 34: 284-298. doi: 10.1016/j.ympev.2004.09.013

Liu H-P, Hershler R (2007) A test of the vicariance hypothesis of western North American freshwater biogeography. Journal of Biogeography 34: 534-548. doi: 10.1111/j.13652699.2006.01611.x

Maciolek JA (1983) Status report: Amargosa toad. Unpublished report to the United States Fish and Wildlife Service, Reno, NV, 21 pp + A1-A28.

Nylander JAA (2004) MrModeltest v2. Program distributed by the author. Evolutionary Biology Centre, Uppsala University.

Reiner SR, Laczniak RJ, DeMeo GA, Smith JL, Elliott PE, Nylund WE, Fridich CJ (2002) Ground-water discharge determined from evapotranspiration, other hydrologic components, and shallow water-level changes, Oasis Valley, Nye County, Nevada. United States Geological Survey Water-Resources Investigation Report 01-4239: 1-65, plates 1-2.

SSI (Systat Software, Inc.) (2004) Systat ${ }^{\oplus}$ for Windows ${ }^{\oplus}$. Richmond, CA.

Stearns REC (1893) Report on the land and fresh-water shells collected in California and Nevada by the Death Valley Exploring Expedition, including a few additional species obtained by Dr. C. Hart Merriam and assistants in parts of the southwestern United States. North American Fauna 7: 269-283.

Stearns REC (1901) The fossil fresh-water shells of the Colorado Desert, their distribution, environment, and variation. Proceedings of the United States National Museum 24: 271-299, plates XIX-XXIV. doi: 10.5479/si.00963801.24-1256.271

Tamura K, Peterson D, Peterson N, Stecher G, Nei M, Kumar S (2011) MEGA5: molecular evolutionary genetics analysis using maximum likelihood, evolutionary distance, and maximum parsimony methods. Molecular Biology and Evolution 28: 2731-2739. doi: $10.1093 / \mathrm{molbev} / \mathrm{msr} 121$

Taylor DW (1985) Evolution of freshwater drainages and molluscs in western North America. In: Smiley CJ, Leviton AJ (Eds) Late Cenozoic History of the Pacific Northwest, Interdisciplinary Studies on the Clarkia Fossil Beds of Northern Idaho. American Association for the Advancement of Science, San Francisco, 265-321.

Taylor DW (1987) Fresh-water molluscs from New Mexico and vicinity. New Mexico Bureau of Mines and Mineral Resources Bulletin 116: 1-50.

USFWS (United States Fish and Wildlife Service) (1976) Proposed endangered or threatened status for 32 U.S. snails. Federal Register 41: 17742-17747.

USFWS (United States Fish and Wildlife Service) (1984) Endangered and threatened wildlife and plants; determination of endangered status and critical habitat for the Amargosa vole. Federal Register 49: 45160-45164.

USFWS (United States Fish and Wildlife Service) (1994) Endangered and threatened wildlife and plants; animal candidate review for listing as endangered or threatened species. Federal Register 59: 58982-59028. 
USFWS (United States Fish and Wildlife Service) (2012a) Endangered and threatened wildlife and plants; determination of endangered status for Three Forks springsnail and threatened status for San Bernardino springsnail throughout their ranges and designation of critical habitat for both species. Federal Register 77: 23060-23092.

USFWS (United States Fish and Wildlife Service) (2012b) Endangered and threatened wildlife and plants; determination of endangered status for the Chupadera springsnail and designation of critical habitat. Federal Register 77: 41088-41106.

USFWS (United States Fish and Wildlife Service) (2012c) Endangered and threatened wildlife and plants; endangered status for six west Texas aquatic invertebrate species and designation of critical habitat. Federal Register 77: 49602-49651. 


\section{Appendix I}

Specimen codes, locality details and GenBank accession numbers for COI and NDI sequences. (doi: 10.3897/zookeys.330.5852.app1) File format: Microsoft Word file (doc).

Copyright notice: This dataset is made available under the Open Database License (http://opendatacommons.org/licenses/odbl/1.0/). The Open Database License $(\mathrm{ODbL})$ is a license agreement intended to allow users to freely share, modify, and use this Dataset while maintaining this same freedom for others, provided that the original source and author(s) are credited.

Citation: Hershler R, Liu H-P, Bradford C (2013) Systematics of a widely distributed western North American springsnail, Pyrgulopsis micrococcus (Caenogastropoda, Hydrobiidae), with descriptions of three new congeners. ZooKeys 330: 55-80. doi: 10.3897/zookeys.330.5852 Specimen codes, locality details and GenBank accession numbers for COI and NDI sequences. doi: 10.3897/zookeys.330.5852.app1

\section{Appendix II}

Genetic distances (maximum composite likelihood) of clades A-F ("Pyrgulopsis micrococcus complex"). (doi: 10.3897/zookeys.330.5852.app2) File format: Microsoft Word file (doc).

Copyright notice: This dataset is made available under the Open Database License (http://opendatacommons.org/licenses/odbl/1.0/). The Open Database License $(\mathrm{ODbL})$ is a license agreement intended to allow users to freely share, modify, and use this Dataset while maintaining this same freedom for others, provided that the original source and author(s) are credited.

Citation: Hershler R, Liu H-P, Bradford C (2013) Systematics of a widely distributed western North American springsnail, Pyrgulopsis micrococcus (Caenogastropoda, Hydrobiidae), with descriptions of three new congeners. ZooKeys 330: 55-80. doi: 10.3897/zookeys.330.5852 Genetic distances (maximum composite likelihood) of clades A-F (“Pyrgulopsis micrococcus complex”). doi: 10.3897/zookeys.330.5852.app2 